\title{
Factors associated with contraceptive failure in Uganda: Analysis of the 2016 Uganda Demographic and Health Survey
}

\section{Short title}

Contraceptive failure in Uganda

\section{Authors}

Ruth Ketty Kisuza ${ }^{1}$, Saviour Kicaber ${ }^{1}$, Derrick Abila Bary ${ }^{1}$, Felix Bongomin ${ }^{2}$, Christopher Orach Garimoi ${ }^{1}$.

\section{Affiliation}

1) College of Health Sciences, Makerere University, Kampala Uganda.

2) Department of Medical Microbiology and Immunology, Faculty of Medicine, Gulu University, Gulu, Uganda

\section{Corresponding author}

Ruth Ketty Kisuza.

College of Health Sciences,

Makerere University, Kampala Uganda

P.O Box 7072

Kampala, Uganda

\section{Abstract}

\section{Introduction}

3 Sustained motivation is essential for effective use of contraceptive methods by women in low- and 4 middle-income countries as many women are likely to abandon use of contraceptives especially 
5 when they continually experience episodes of failure. We aimed to determine contraceptive failure

6 rates and associated factors among Ugandan women using data from the 2016 Uganda

7 Demographic Health Survey (UDHS).

8 Methods

9 We analyzed data collected by the UDHS conducted in Uganda 2016. All eligible women aged 15

10 to 49 years at the time of the survey were enrolled. Discontinuation of contraceptive use due to

11 failure within a 5-year period preceding the survey was the dependent variable.

\section{Results}

13 A total of 18,505 women were included in this study, $70.8 \%(n=5153)$ lived in rural areas while $56.9 \%(\mathrm{n}=5153)$ owned a mobile phone. The mean age of the women was 29.6years (SD 7.6). The

15 overall prevalence of contraceptive failure was 5.6\%, and was higher (7.8\%) among women aged

$1738 \%$ lower in women who had an informed choice on contraceptives compared to those who didn't [Adjusted Odd ratio, 0.62; 95\% confidence interval, $0.50-0.77 ; \mathrm{p}<0.001$ ].

\section{Conclusion}

20 The burden of contraceptive failure among women of reproductive age in Uganda is substantial

21 and significantly varied by socio-demographic characteristics.

22 Key words: Contraceptive failure, Uganda, Demographic Health Survey (DHS) and Sociodemographic Characteristics.

\section{Introduction}

26 Improving access to family planning (FP) services is fundamental to achieving the Sustainable

27 Development Goals (SDGs) because it is strongly related with women's and children's health, 
medRxiv preprint doi: https://doi.org/10.1101/2022.02.09.22270716; this version posted February 9, 2022. The copyright holder for this preprint (which was not certified by peer review) is the author/funder, who has granted medRxiv a license to display the preprint in perpetuity.

It is made available under a CC-BY 4.0 International license.

28 poverty reduction, education, gender equality, and human rights. Access to family planning

29 contributes up to a 44\% reduction in maternal deaths [1]. Since most unplanned pregnancies and

30 abortions occur in women who were either not using contraception or not using it consistently.

31 Greater access to contraception and more consistent use of contraception are crucial in the

32 reduction of unplanned pregnancies and abortions.

34 The reasons for discontinuation of contraceptives can be grouped by whether they represent

35 discontinuations due to reduced need for contraception (not in need) or discontinuations while

36 women were presumably still exposed to the risk of pregnancy and did not want to become

37 pregnant (in need). Among discontinuations that are not in need, the most common reason given

38 is wanting to become pregnant. Among in-need discontinuations, the most common reasons given

39 are women that become pregnant while using the method (contraceptive failure) or those that

40 stopped using the method because of side effects[2].

41

42 High rates of discontinuation for reasons other than the desire for pregnancy are problematic

43 because of their association with several negative reproductive health outcomes. In countries with

44 moderate-high contraceptive prevalence most of the unintended pregnancy is the result of

45 contraceptive discontinuation or failure. Several studies have associated contraceptive

46 discontinuation for reasons other than the desire to become pregnant with unmet need for

47 contraception and induced abortion ([2-4]. Unintended pregnancy has been associated with

48 increased risk of maternal morbidity, health behaviors during pregnancy that are associated with

49 adverse maternal health, and adverse fetal, infant and child health outcomes [5] Additionally, 
medRxiv preprint doi: https://doi.org/10.1101/2022.02.09.22270716; this version posted February 9, 2022. The copyright holder for this preprint (which was not certified by peer review) is the author/funder, who has granted medRxiv a license to display the preprint in perpetuity.

It is made available under a CC-BY 4.0 International license.

50 unintended pregnancy has been associated with negative psychological effects on women and their

51 children[6].

52

53 In the developing world, 74 million unintended pregnancies occur annually, and nearly a third

$5430 \%$, are due to contraceptive failure among women using some type of contraceptive method

55 (whether traditional or modern). This includes both method-related failures (i.e., failure of a

56 method to work as expected) and user-related failures (i.e., failure due to incorrect or inconsistent

57 use of a method)[7].

58

59 Sustained motivation is essential for effective use of contraceptive methods by women in low- and

60 middle-income countries as many women are likely to abandon use of contraceptives especially

61 when they continually experience episodes of failure. Assessing failure rates among demographic

62 and socioeconomic groups is important to inform efforts to improve contraceptive information,

63 services and use, and to minimize contraceptive failures. Additionally, detailed information on

64 contraceptive failure rates is critical to inform improvements in provision of contraceptive

65 information, supplies and services, which can help women and couples to use methods correctly

66 and consistently. This study aimed to determine the contraceptive failure rates and the factors

67 associated with contraceptive failure among Ugandan women using the 2016 Uganda

68

Demographic Health Survey (UDHS) data.

69

70 


\section{Methods}

\section{Study design and setting:}

73 We analyzed data collected during the UDHS conducted in 2016. "The Demographic and Health

74 Surveys (DHS) are internationally comparable household surveys that collect information on

75 demographic, socioeconomic, and health-related variables among nationally representative

76 samples of households in developing countries. Details of the DHS sampling design and strategies

77 are described elsewhere"[8].

\section{Study population}

79 In this study, we used the women recode file which included women aged 15 to 49 years at the

80 time of the survey. We included all the women in the dataset into our analysis.

\section{Study variables}

82 Discontinuation of contraceptive use due to failure within a five-year period preceding the survey

83 was the dependent variable. The DHS does not have a variable that records discontinuation of

84 contraceptive use due to failure. This was derived from the variable v360 that records the various

85 reasons for discontinuation such as pregnancy, wanting to become pregnant, side effects and

86 wanting a more effective method. In this study, a woman becoming pregnant while on

87 contraceptives was termed as a method failure (exposure) while discontinuation due to another

88 reason was termed as non-exposure.

89 The independent variables used in the study included (1) age, (2) type of place of residence (rural

90 vs urban), (3) wealth index, (4) education attainment, (5) phone ownership, (6) internet use, (7)

91 number of children delivered, (8) Women's participation in decision-making for using

92 contraceptives, and (9) work status. We also included the variable on the method of contraception that was discontinued over the 5-year period preceding the survey. 
medRxiv preprint doi: https://doi.org/10.1101/2022.02.09.22270716; this version posted February 9, 2022. The copyright holder for this preprint (which was not certified by peer review) is the author/funder, who has granted medRxiv a license to display the preprint in perpetuity.

It is made available under a CC-BY 4.0 International license.

\section{Data management}

95 The data used were obtained after receiving permission from the DHS program website. The dataset for the Uganda DHS 2016 was then downloaded from the DHS program website. The

97 women's recode file readable by STATA version 13 was selected for use.

To calculate failure rate, we created a new variable for which the denominator would be the total of the number of women who were currently using contraceptives at time of survey interview and the number of women who were not using at the time of survey because they had discontinued.

101 The number of women who were currently using contraceptives at time of survey were obtained by converting variable v312 in the DHS into a binary variable (currently use vs. non-currently using). The number of women who were not using contraceptives at the time of the survey because they discontinued was obtained in the following way. First, we created a new variable for only women who were not currently using contraceptives at the time of the survey. This was recorded into a binary variable with 0 if a woman had not discontinued contraceptives and 1 if a woman discontinued contraceptives. The latter was used to obtain the number of women who were not using contraceptives at the time of the survey because they discontinued.

109 The numerator for calculating contraceptive failure rate was obtained by converting DHS variable

110 v360 (reason for discontinuation) into a binary variable with yes (1) if reason was

111 failure/pregnancy and no (0) if other reason for discontinuation. The numerator considered was

112 the number of women who discontinued due failure.

\section{Data analysis}

114 All the analysis in this study was performed in STATA13 and each survey dataset was analyzed 115 separately (StataCorp LP 2013). Weighting was performed for all the descriptive statistics using the weight variable (v005) after dividing it by 1,000,000. 


$$
\text { weight }=\frac{\mathrm{V} 005}{1,000,000}
$$

118 For the regression analysis in STATA13, weighting was also performed using the Primary

119 Sampling Unit (PSU) and Strata as the variables v021 and v022 respectively in the DHS survey

120 datasets for the surveys and weight (wgt) calculated previously. The STATA13 code below was

121 used to apply weights before calculating means, standard deviation and 95\% confidence intervals

122 of continuous variables and regression analysis.

123 svyset v021 [pw = wgt], strata(v022) single unit(centered)

124 where pw is the probability weight (sampling weight), psu is primary sampling unit, v021 is

125 variable for the primary sampling unit, and v022 is the variable in the DHS that indicates the strata

126 used in the DHS surveys.

\section{Summary statistics}

128 For the categorical variables, we summarized them as weighted proportions. Variables that were 129 numeric like age were summarized as weighted means reporting standard deviations and 95\% 130 confidence intervals.

\section{Regression analysis}

132 We used bivariate and multivariate logistic regression to determine the association between demographic characteristics and contraceptive failure among women. We reported crude odds

134 ratios, adjusted odds ratios, p-values, and 95\% confidence intervals. First, bivariate logistic 135 regression was used to determine the association between contraceptive failure and the women's 136 demographic characteristics. Then, multivariate logistic regression was used including the 137 variables that had a p-value of less than 0.200 i.e. (1) age group (2) number of children a woman 138 has, and (3) Frequency of using the internet last month. 
medRxiv preprint doi: https://doi.org/10.1101/2022.02.09.22270716; this version posted February 9, 2022. The copyright holder for this preprint (which was not certified by peer review) is the author/funder, who has granted medRxiv a license to display the preprint in perpetuity.

It is made available under a CC-BY 4.0 International license.

\section{Quality control}

142 To ensure that we are working with the correct DHS dataset for a given year, a test analysis was 143 performed to try to replicate the tables in the respective DHS survey reports for the various years.

144 We created a frequency distribution table for the variable of type of place of residence for the

145 different years in STATA13 and compared the result with what was reported in the respective 146 DHS survey report. All results from this test analysis matched those in the DHS survey reports.

147 Results

148 Characteristics of the women

149 Table 1 shows the socio-demographic characteristics of the sample of women aged 15-49 who 150 had used and never used a contraceptive method within the five years before the 2016 UDHS. 151 Among contraceptive users $(n=8000), 23.4 \%$ were between age $25-29$ years, only $7.6 \%(n=691)$ 152 were adolescents (age 15-19 years) and 4.4\% $(\mathrm{n}=401)$ were above age $45-49$ years. Only $7.8 \%$ had 153 no formal education. Majority $(80.3 \%)$ of the women were working and about $70.7 \%$ lived in rural 154 areas of Uganda. About 28.9\% $(n=2573)$ of the women belonged to the highest wealth quintile. 155 Majority of women who used contraceptives had never been exposed to the internet in the last 12 months $(89.0 \%), 9.6 \%(n=873)$ had used the internet in the last 12 months. Additionally, 56.8\%

$157(n=5153)$ of the women who used contraceptives owned a mobile phone. Among cultural factors, 158 about two-thirds of the women made a joint decision with their partners on contraceptive use. $15941.8 \%(n=3788)$ of the women using contraceptives made an informed choice while 58.2\% $160(n=5273)$ of the women did not make an informed choice. The most common methods used and 161 discontinued in the past 5 years were injectable $(54.8 \%)$, followed by implants $(10.5 \%)$. 
medRxiv preprint doi: https://doi.org/10.1101/2022.02.09.22270716; this version posted February 9, 2022. The copyright holder for this preprint (which was not certified by peer review) is the author/funder, who has granted medRxiv a license to display the preprint in perpetuity.

It is made available under a CC-BY 4.0 International license.

Table 1: Distribution of method related, demographic, socio economic and cultural characteristics of women by status of contraceptive use.

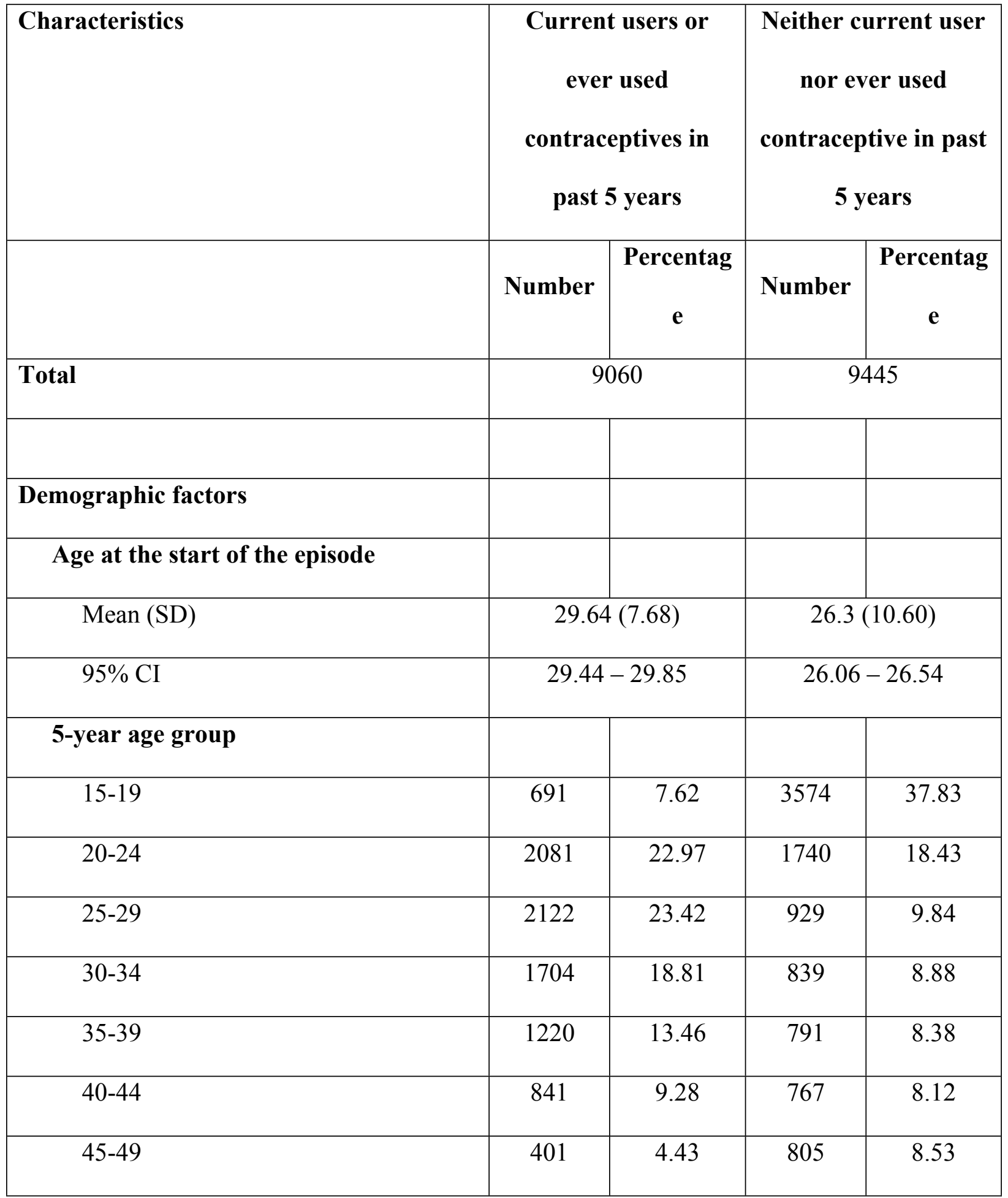




\begin{tabular}{|c|c|c|c|c|}
\hline $\begin{array}{l}\text { Parity (living children at the end of the } \\
\text { episode) }\end{array}$ & & & & \\
\hline Mean (SD) & \multicolumn{2}{|c|}{$3.78(2.66)$} & \multicolumn{2}{|c|}{$2.41(3.11)$} \\
\hline $95 \% \mathrm{CI}$ & \multicolumn{2}{|c|}{$3.7-3.87$} & \multicolumn{2}{|c|}{$2.33-2.49$} \\
\hline \multicolumn{5}{|l|}{ Socioeconomic factors } \\
\hline \multicolumn{5}{|l|}{ Internet use in the past year } \\
\hline Never & 8067 & 89.03 & 8624 & 91.31 \\
\hline Yes, last 12 months & 873 & 9.64 & 723 & 7.66 \\
\hline Yes, before last 12 months & 120 & 1.33 & 98 & 1.04 \\
\hline \multicolumn{5}{|l|}{ Frequency of using internet last month } \\
\hline Not at all & 8776 & 92.70 & 8230 & 90.83 \\
\hline Less than once a week & 127 & 1.35 & 119 & 1.32 \\
\hline At least once a week & 187 & 1.98 & 285 & 3.14 \\
\hline Almost every day & 376 & 3.98 & 426 & 4.71 \\
\hline \multicolumn{5}{|l|}{ Mobile phone ownership } \\
\hline No & 3907 & 43.12 & 6170 & 65.32 \\
\hline Yes & 5153 & 56.88 & 3275 & 34.68 \\
\hline \multicolumn{5}{|l|}{ Women's education attainment } \\
\hline No education & 678 & 7.48 & 1103 & 11.68 \\
\hline Incomplete primary & 3783 & 41.76 & 4474 & 47.36 \\
\hline Complete Primary & 1291 & 14.26 & 1081 & 11.45 \\
\hline Incomplete secondary & 2251 & 24.85 & 2093 & 22.16 \\
\hline Complete secondary & 169 & 1.87 & 125 & 1.32 \\
\hline
\end{tabular}




\begin{tabular}{|c|c|c|c|c|}
\hline Higher & 887 & 9.79 & 569 & 6.02 \\
\hline \multicolumn{5}{|l|}{ Currently/formerly/never in union } \\
\hline Never in union & 863 & 9.52 & 3920 & 41.50 \\
\hline Currently in union/living with a man & 6939 & 76.58 & 4284 & 45.36 \\
\hline Formerly in union/living with a man & 1259 & 13.89 & 1241 & 13.14 \\
\hline \multicolumn{5}{|l|}{$\begin{array}{ll}\text { Partners/Husbands } & \text { education } \\
\text { attainment }(n=11,223) & \end{array}$} \\
\hline No formal education & 287 & 4.13 & 425 & 9.93 \\
\hline Incomplete primary & 2191 & 31.58 & 1609 & 37.55 \\
\hline Complete Primary & 1233 & 17.77 & 798 & 18.62 \\
\hline Incomplete secondary & 1744 & 25.15 & 861 & 20.11 \\
\hline Complete secondary & 304 & 4.39 & 101 & 2.35 \\
\hline Higher & 976 & 14.07 & 382 & 8.91 \\
\hline Do not know & 202 & 2.91 & 109 & 2.54 \\
\hline \multicolumn{5}{|l|}{ Husband's/partner's age } \\
\hline $15-19$ & 18 & 0.26 & 56 & 1.31 \\
\hline $20-24$ & 503 & 7.25 & 621 & 14.49 \\
\hline $25-29$ & 1220 & 17.59 & 685 & 15.98 \\
\hline $30-34$ & 1433 & 20.65 & 650 & 15.16 \\
\hline $35-39$ & 1256 & 18.11 & 581 & 13.56 \\
\hline $40-44$ & 1055 & 15.20 & 488 & 11.39 \\
\hline $45-49$ & 759 & 10.94 & 490 & 11.44 \\
\hline $50-54$ & 423 & 6.10 & 380 & 8.88 \\
\hline
\end{tabular}




\begin{tabular}{|c|c|c|c|c|}
\hline $55-59$ & 135 & 1.95 & 140 & 3.27 \\
\hline 60 years or more & 136 & 1.96 & 194 & 4.52 \\
\hline \multicolumn{5}{|l|}{ Work status } \\
\hline Not currently employed & 1784 & 19.69 & 3201 & 33.90 \\
\hline Currently employed & 7276 & 80.31 & 6243 & 66.10 \\
\hline \multicolumn{5}{|l|}{ Place of residence } \\
\hline Urban & 2650 & 29.24 & 2293 & 24.28 \\
\hline Rural & 6411 & 70.76 & 7152 & 75.72 \\
\hline \multicolumn{5}{|c|}{ Household wealth index quintile } \\
\hline Lowest & 1205 & 13.30 & 2041 & 21.61 \\
\hline Second & 1600 & 17.67 & 1796 & 19.01 \\
\hline Middle & 1725 & 19.04 & 1734 & 18.36 \\
\hline Fourth & 1956 & 21.59 & 1727 & 18.28 \\
\hline Highest & 2573 & 28.39 & 2147 & 22.73 \\
\hline \multicolumn{5}{|l|}{ Cultural factors } \\
\hline \multicolumn{5}{|c|}{$\begin{array}{l}\text { Women participation in decision- } \\
\text { making for using contraceptives }\end{array}$} \\
\hline Mainly woman & 1340 & 30.64 & - & - \\
\hline Mainly husband/partner & 312 & 7.13 & - & - \\
\hline Joint decision & 2710 & 61.98 & - & - \\
\hline Other & 11 & 0.25 & - & - \\
\hline \multicolumn{5}{|l|}{ Informed choice } \\
\hline No & 5273 & 58.20 & - & - \\
\hline
\end{tabular}




\begin{tabular}{|c|c|c|c|c|}
\hline Yes & 3788 & 41.80 & - & - \\
\hline \multicolumn{5}{|l|}{ Method-related factors } \\
\hline \multicolumn{5}{|c|}{ Contraceptive method discontinued } \\
\hline Pill & 553 & 9.64 & - & - \\
\hline IUD & 161 & 2.80 & - & - \\
\hline Injectable & 3150 & 54.85 & - & - \\
\hline Male condom & 444 & 7.72 & - & - \\
\hline Male sterilization & 1 & 0.01 & - & - \\
\hline Periodic abstinence & 188 & 3.27 & - & - \\
\hline Withdraw & 371 & 6.46 & - & - \\
\hline Other traditional & 42 & 0.73 & - & - \\
\hline Implants/Norplant & 605 & 10.54 & - & - \\
\hline Lactational amenorrhea (lam) & 155 & 2.71 & - & - \\
\hline Female condom & 2 & 0.03 & - & - \\
\hline Emergency contraception & 22 & 0.38 & - & - \\
\hline Other modern method & 15 & 0.26 & - & - \\
\hline Standard days method (sdm) & 35 & 0.61 & - & - \\
\hline & & & & \\
\hline
\end{tabular}

$\mathrm{IUD}=$ intrauterine contraceptive device

167 As shown in Table 2, the prevalence of contraceptive failure among Ugandan women aged 15-49

168 years during the 5-year period by demographic, socio economic and cultural factors was 5.6\% 
medRxiv preprint doi: https://doi.org/10.1101/2022.02.09.22270716; this version posted February 9, 2022. The copyright holder for this preprint (which was not certified by peer review) is the author/funder, who has granted medRxiv a license to display the preprint in perpetuity.

It is made available under a CC-BY 4.0 International license.

$169(\mathrm{n}=506)$. Among the demographic factors, failure rate $7.83 \%(\mathrm{n}=157)$ was prevalent in women

170

171

172

173

174

175

176

177

178

179

180 aged 25-29 years and having five or more children (6.35\%). For socioeconomic factors, the highest prevalence rates were seen in women who used internet almost every day in the one month $(7.78 \%$, $\mathrm{n}=426)$, owned a mobile phone $(5.72 \%, \mathrm{n}=294)$, completed higher education $(7.09 \%, \mathrm{n}=63)$, unemployed $(5.83 \%, \mathrm{n}=104)$ and lived in a rural area $(5.70 \%, \mathrm{n}=364)$.

Among cultural factors, women who had a joint decision for contraceptive use with their partner had the highest failure of the $4.95 \%(n=134)$. Women who made an informed choice on contraceptive use had low contraceptive failure rates $(4.43 \%, \mathrm{n}=168)$ as compared to those who didn't make an informed choice $(6.41 \%, \mathrm{n}=338)$.

Table 2: Prevalence of contraceptive failure among Ugandan women aged 15-49 years during a five-year period by demographic, socioeconomic, and cultural factors.

\begin{tabular}{|c|c|c|c|}
\hline & \multicolumn{2}{|c|}{ 5-year contraceptive } \\
& Number & \multicolumn{2}{|c|}{} \\
& using & No. disc. & Percentage \\
& contraceptiv & & \\
\hline Demographic characteristics & es & 5.58 \\
\hline Total & 9061 & 506 & \\
\hline Demographic factors & & & \\
\hline 5-year age group & 690 & 19 & 2.82 \\
\hline $15-19$ & & & \\
\hline
\end{tabular}




\begin{tabular}{|c|c|c|c|}
\hline $20-24$ & 2081 & 105 & 5.02 \\
\hline $25-29$ & 2122 & 157 & 7.38 \\
\hline $30-34$ & 1704 & 105 & 6.19 \\
\hline $35-39$ & 1220 & 66 & 5.40 \\
\hline $40-44$ & 841 & 41 & 4.84 \\
\hline $45-49$ & 401 & 13 & 3.22 \\
\hline \multicolumn{4}{|c|}{ Parity (total children ever born) } \\
\hline One & 1298 & 58 & 4.46 \\
\hline Two & 1489 & 73 & 4.88 \\
\hline Three & 1312 & 77 & 5.88 \\
\hline Four & 1164 & 73 & 6.25 \\
\hline Five or more & 3119 & 198 & 6.35 \\
\hline \multicolumn{4}{|l|}{ Socioeconomic factors } \\
\hline \multicolumn{4}{|l|}{ Internet use in the past year } \\
\hline Never & 8067 & 440 & 5.45 \\
\hline Yes, last 12 months & 873 & 57 & 6.58 \\
\hline Yes, before last 12 months & 120 & 8 & 6.79 \\
\hline \multicolumn{4}{|c|}{ Frequency of using internet last month } \\
\hline Not at all & 8230 & 450 & 5.47 \\
\hline Less than once a week & 119 & 6 & 4.67 \\
\hline At least once a week & 285 & 17 & 5.91 \\
\hline Almost every day & 426 & 33 & 7.78 \\
\hline
\end{tabular}




\begin{tabular}{|c|c|c|c|}
\hline Mobile phone ownership & & & \\
\hline No & 3907 & 211 & 5.40 \\
\hline Yes & 5153 & 295 & 5.72 \\
\hline \multicolumn{4}{|l|}{ Education } \\
\hline No education & 678 & 35 & 5.10 \\
\hline Incomplete primary & 3783 & 194 & 5.12 \\
\hline Complete Primary & 1292 & 66 & 5.10 \\
\hline Incomplete secondary & 2251 & 136 & 6.05 \\
\hline Complete secondary & 169 & 12 & 7.14 \\
\hline Higher & 887 & 63 & 7.09 \\
\hline \multicolumn{4}{|l|}{ Currently/formerly/never in union } \\
\hline Never in union & 863 & 24 & 2.74 \\
\hline Currently in union/living with a man & 6939 & 431 & 6.21 \\
\hline Formerly in union/living with a man & 1259 & 51 & 4.03 \\
\hline $\begin{array}{l}\text { Partners/Husbands education attainn } \\
=11,223)\end{array}$ & & & \\
\hline No education & 287 & 23 & 8.12 \\
\hline Incomplete primary & 2191 & 122 & 5.56 \\
\hline Complete Primary & 1233 & 76 & 61.6 \\
\hline Incomplete secondary & 1745 & 104 & 5.94 \\
\hline Complete secondary & 304 & 27 & 8.98 \\
\hline Higher & 976 & 63 & 6.41 \\
\hline Do not know & 202 & 16 & 8.15 \\
\hline
\end{tabular}




\begin{tabular}{|c|c|c|c|}
\hline Husband's/partner's age & & & \\
\hline $15-19$ & 18 & 0 & 0 \\
\hline $20-24$ & 503 & 32 & 6.28 \\
\hline $25-29$ & 1220 & 67 & 5.50 \\
\hline $30-34$ & 1433 & 106 & 7.38 \\
\hline $35-39$ & 1256 & 91 & 7.27 \\
\hline $40-44$ & 1055 & 46 & 4.38 \\
\hline $45-49$ & 759 & 56 & 7.39 \\
\hline $50-54$ & 423 & 21 & 4.97 \\
\hline $55-59$ & 135 & 2 & 1.73 \\
\hline 60 years or more & 136 & 10 & 7.09 \\
\hline \multicolumn{4}{|l|}{ Work status } \\
\hline Not currently employed & 1784 & 104 & 5.83 \\
\hline Currently employed & 7277 & 401 & 5.52 \\
\hline \multicolumn{4}{|l|}{ Place of residence } \\
\hline Urban & 2650 & 140 & 5.30 \\
\hline Rural & 6411 & 365 & 5.70 \\
\hline \multicolumn{4}{|c|}{ Household wealth index quintile } \\
\hline Lowest & 1205 & 64 & 5.31 \\
\hline Second & 1601 & 85 & 5.29 \\
\hline Middle & 1725 & 97 & 5.60 \\
\hline Fourth & 1956 & 116 & 5.93 \\
\hline Highest & 2573 & 144 & 5.60 \\
\hline
\end{tabular}




\begin{tabular}{|l|c|c|c|}
\hline Cultural factors & & & \\
\hline Women participation in decision-making for & & & \\
\hline using contraceptives & & 64 & 4.81 \\
\hline Mainly woman & 1340 & 9 & 3.00 \\
\hline Mainly husband/partner & 311 & 134 & 4.95 \\
\hline Joint decision & 2710 & - & - \\
\hline Other & - & & \\
\hline Informed choice & & & \\
\hline No & 5273 & 338 & 6.41 \\
\hline Yes & 3788 & 168 & 4.43 \\
\hline
\end{tabular}

Table 3 shows the contraceptive failure rates in Uganda by method used. Generally traditional methods had the highest failure rates. Of this, withdrawal $(36.30 \%, n=134)$ was the single most common reason for failure. For modern contraceptive methods, short term methods like emergency contraception $(20.85 \%, n=5)$ and pills $(10.68 \%, n=59)$ had higher failure rates when compared to

187 very low failure rates.

Table 3: Contraceptive failure rates in Uganda by methods used.

\begin{tabular}{|l|c|c|c|}
\hline Method-related factors & & & \\
\hline Contraceptive method & Number who used & Number & Percentage (\%) \\
discontinued & the method & failed & \\
\hline
\end{tabular}


medRxiv preprint doi: https://doi.org/10.1101/2022.02.09.22270716; this version posted February 9, 2022. The copyright holder for this preprint (which was not certified by peer review) is the author/funder, who has granted medRxiv a license to display the preprint in perpetuity.

It is made available under a CC-BY 4.0 International license.

\begin{tabular}{|c|c|c|c|}
\hline Pill & 553 & 59 & 10.68 \\
\hline IUD & 161 & 3 & 1.62 \\
\hline Injectable & 3150 & 140 & 4.43 \\
\hline Male condom & 444 & 31 & 6.93 \\
\hline Male sterilization & 1 & 0 & 0 \\
\hline Periodic abstinence & 188 & 65 & 34.87 \\
\hline Withdraw & 371 & 134 & 36.30 \\
\hline Other traditional & 41 & 19 & 44.78 \\
\hline Implants/Norplant & 605 & 15 & 2.60 \\
\hline $\begin{array}{l}\text { Lactational amenorrhea } \\
\text { (lam) }\end{array}$ & 155 & 20 & 12.69 \\
\hline Female condom & 2 & 1 & 50.00 \\
\hline Emergency contraception & 22 & 5 & 20.85 \\
\hline Other modern method & 15 & 2 & 13.43 \\
\hline Standard days method (sdm) & 35 & 12 & 34.17 \\
\hline
\end{tabular}


192 From the multivariate analysis, women aged 45 to 49 years [Adjusted Odds ratio (aOR), 0.33 ; 95\% confidence interval (95CI), 0.12 $1930.94 ; \mathrm{p}$ value $=0.038$ ] were less likely to experience a contraceptive failure compared to those aged 15 to 19 years. Women who had an informed choice on contraceptives were less likely to experience a failure [aOR, 0.62; 95CI, $0.50-0.77 ; \mathrm{p}$-value $<0.001]$ compared to those who did not have informed choice (Table 4).

197 Table 4: Factors associated with contraceptive failure among women in Uganda.

\begin{tabular}{|c|c|c|c|c|}
\hline & \multicolumn{2}{|c|}{ Bivariate analysis } & \multicolumn{2}{c|}{ Multivariate analysis } \\
\hline Demographic characteristics & $\begin{array}{c}\text { Crude Odds Ratio } \\
\mathbf{( 9 5 \%} \mathbf{C I})\end{array}$ & P - value & $\begin{array}{c}\text { Adjusted Odds } \\
\text { Ratio } \\
\text { (95\% CI) }\end{array}$ & P - value \\
\hline Demographic factors & & & & \\
\hline 5-year age group & & & & \\
\hline $15-19$ & 1 & & 1 & \\
\hline $20-24$ & $1.73(1.04-2.86)$ & 0.034 & $1.28(0.66-2.49)$ & 0.464 \\
\hline $25-29$ & $2.61(1.59-4.27)$ & $<0.001$ & $1.56(0.75-3.25)$ & 0.234 \\
\hline
\end{tabular}




\begin{tabular}{|c|c|c|c|c|}
\hline $30-34$ & $2.19(1.32-3.63)$ & 0.002 & $0.98(0.45-2.16)$ & 0.967 \\
\hline $35-39$ & $2.05(1.22-3.46)$ & 0.007 & $0.83(0.36-1.90)$ & 0.664 \\
\hline $40-44$ & $1.56(0.88-2.76)$ & 0.127 & $0.53(0.22-1.3)$ & 0.166 \\
\hline $45-49$ & $1.05(0.496-2.211)$ & 0.903 & $0.33(0.12-0.94)$ & 0.038 \\
\hline \multicolumn{5}{|c|}{ Parity (total children ever born) } \\
\hline No child & 1 & & 1 & \\
\hline One child & $1.58(0.93-2.66)$ & 0.089 & $0.68(0.34-1.36)$ & 0.277 \\
\hline Two children & $1.45(0.87-2.44)$ & 0.157 & $0.66(0.33-1.32)$ & 0.239 \\
\hline Three children & $1.80(1.08-3.01)$ & 0.024 & $0.79(0.39-1.62)$ & 0.522 \\
\hline Four children & $1.895(1.131-3.175)$ & 0.015 & $0.92(0.43-1.93)$ & 0.818 \\
\hline Five or more children & $2.16(1.35-3.47)$ & 0.001 & $1.70(0.80-3.61)$ & 0.166 \\
\hline \multicolumn{5}{|l|}{ Socioeconomic factors } \\
\hline \multicolumn{5}{|l|}{ Internet use in the past year } \\
\hline Never & 1 & & - & \\
\hline Yes, last 12 months & $1.08(0.78-1.49)$ & 0.637 & - & - \\
\hline Yes, before last 12 months & $1.42(0.70-2.88)$ & 0.329 & - & - \\
\hline
\end{tabular}




\begin{tabular}{|l|c|c|c|c|}
\hline Frequency of using internet last month & & & & \\
\hline Not at all & 1 & & - & \\
\hline Less than once a week & $0.73(0.28-1.88)$ & 0.512 & - & - \\
\hline At least once a week & $1.46(0.89-2.398)$ & 0.136 & - & - \\
\hline Almost every day & $1.01(0.64-1.59)$ & 0.971 & - & \\
\hline Mobile phone ownership & & & & \\
\hline No & 1 & & - & - \\
\hline Yes & $0.99(0.82-1.19)$ & 0.918 & - & \\
\hline Education & & & & - \\
\hline No education & $1.01(0.698-1.454)$ & 0.969 & & - \\
\hline Incomplete primary & $1.09(0.72-1.65)$ & 0.678 & & - \\
\hline Complete Primary & $1.18(0.799-1.73)$ & 0.410 & & - \\
\hline Incomplete secondary & $1.32(0.63-2.77)$ & 0.461 & & - \\
\hline Complete secondary & $1.20(0.77-1.89)$ & 0.416 & & - \\
\hline Higher & & & - & - \\
\hline Currently/formerly/never in union & & & - \\
\hline
\end{tabular}




\begin{tabular}{|c|c|c|c|c|}
\hline Never in union & 1 & & & \\
\hline Currently in union/living with a man & $2.15(1.41-3.28)$ & $<0.001$ & - & \\
\hline Formerly in union/living with a man & $1.39(0.84-2.30)$ & 0.205 & - & \\
\hline \multicolumn{5}{|c|}{ Partners/Husbands education attainment $(n=$} \\
\hline No education & 1 & & & \\
\hline Incomplete primary & $0.66(0.42-1.04)$ & 0.074 & - & - \\
\hline Complete Primary & $0.76(0.47-1.22)$ & 0.253 & - & - \\
\hline Incomplete secondary & $0.68(0.42-1.09)$ & 0.110 & - & - \\
\hline Complete secondary & $1.06(0.58-1.94)$ & 0.843 & - & - \\
\hline Higher & $0.76(0.46-1.25)$ & 0.278 & - & - \\
\hline Do not know & $0.90(0.45-1.81)$ & 0.772 & - & - \\
\hline \multicolumn{5}{|l|}{ Husband's/partner's age } \\
\hline $15-19$ & 1 & & 1 & \\
\hline $20-24$ & $2.93(0.17-49.43)$ & 0.456 & $2.75(0.16-47.27)$ & 0.486 \\
\hline $25-29$ & $2.69(0.16-44.71)$ & 0.491 & $1.99(0.11-34.47)$ & 0.638 \\
\hline
\end{tabular}




\begin{tabular}{|c|c|c|c|c|}
\hline $30-34$ & $3.81(0.23-63.21)$ & 0.350 & $2.43(0.14-42.32)$ & 0.543 \\
\hline $35-39$ & $3.39(0.20-56.34)$ & 0.394 & $2.26(0.13-39.68)$ & 0.577 \\
\hline $40-44$ & $2.49(0.15-41.64)$ & 0.524 & $1.75(0.098-31.00)$ & 0.704 \\
\hline $45-49$ & $3.42(0.21-57.14)$ & 0.392 & $2.78(0.16-49.49)$ & 0.487 \\
\hline $50-54$ & $2.66(0.16-45.18)$ & 0.499 & $2.5(0.14-45.56)$ & 0.537 \\
\hline $55-59$ & $1.4(0.07-27.98)$ & 0.826 & $1.46(0.07-31.20)$ & 0.810 \\
\hline 60 years or more & $3.34(0.19-59.89)$ & 0.412 & $3.02(0.16-57.77)$ & 0.463 \\
\hline \multicolumn{5}{|l|}{ Work status } \\
\hline Not currently employed & 1 & & - & \\
\hline Currently employed & $1.02(0.80-1.31)$ & 0.849 & - & - \\
\hline \multicolumn{5}{|l|}{ Place of residence } \\
\hline Urban & 1 & & 1 & \\
\hline Rural & $1.03(0.83-1.27)$ & 0.803 & $1.04(0.8-1.4)$ & 0.789 \\
\hline \multicolumn{5}{|c|}{ Household wealth index quintile } \\
\hline Lowest & 1 & & - & \\
\hline Second & $1.06(0.78-1.45)$ & 0.712 & - & - \\
\hline
\end{tabular}




\begin{tabular}{|l|c|c|c|c|}
\hline Middle & $0.98(0.71-1.34)$ & 0.901 & - & - \\
\hline Fourth & $1.05(0.77-1.43)$ & 0.747 & - & - \\
\hline Highest & $0.95(0.70-1.29)$ & 0.760 & - & - \\
\hline Cultural factors & & & & \\
\hline Women participation in decision-making for using contraceptives & & & \\
\hline Mainly woman. & 1 & & - & - \\
\hline Mainly husband/partner & $0.75(0.38-1.46)$ & 0.399 & - & - \\
\hline Joint decision & $1.01(0.73-1.399)$ & 0.933 & - & - \\
\hline Other & $1.27(0.07-22.20)$ & 0.872 & - & $<$ \\
\hline Informed choice & & & & \\
\hline No & & & & - \\
\hline Yes & $0.65(0.53-0.79)$ & $<0.001$ & $0.62(0.50-0.77)$ & $<\mathbf{0 . 0 0 1}$ \\
\hline
\end{tabular}


medRxiv preprint doi: https://doi.org/10.1101/2022.02.09.22270716; this version posted February 9, 2022. The copyright holder for this preprint (which was not certified by peer review) is the author/funder, who has granted medRxiv a license to display the preprint in perpetuity.

It is made available under a CC-BY 4.0 International license.

\section{Discussion}

In this study, we aimed to establish and describe the prevalence of contraceptive failure amongst women of reproductive age in Uganda. We used data from the demographic and health survey conducted in Uganda in 2016. From this study, it is evident that the prevalence of contraceptive failure varies by age, type of place of residence, phone ownership, education attainment and wealth index. The highest failure rates were seen in women who use periodic abstinence and withdraw as methods of contraception. Finally, women who had delivered four or more children were more likely to experience contraceptive failure when compared with those who had not delivered any child.

The study shows that women with access to the internet were more likely to experience contraceptive failure compared to women with no access to the internet. This could be because the women with access to the internet can easily access information on the different contraceptive methods and are therefore more likely to stop or switch to better contraceptive methods when they experience side effects [9]. Additionally, women who were provided with adequate information about a particular contraceptive method and given an opportunity to make an informed choice were less likely to experience contraceptive failure than women who did not make an informed choice. This could be because a woman who made an informed choice had adequate information about the contraceptive method of her choice and was able to effectively deal with its side effects without necessarily halting contraceptive use $[10,11]$. This finding suggests that more time should be dedicated by clinicians to providing information on contraceptives to women before use.

In this study, failure rates by contraceptive methods were highest in women who used traditional methods than in those who used modern contraceptive methods. For modern contraceptive 
medRxiv preprint doi: https://doi.org/10.1101/2022.02.09.22270716; this version posted February 9, 2022. The copyright holder for this preprint (which was not certified by peer review) is the author/funder, who has granted medRxiv a license to display the preprint in perpetuity.

It is made available under a CC-BY 4.0 International license.

222

223

224

225

226

227

228

229

230

231

232

233

234

235

236

237

238

239

240

241

242

243

244

methods, women using short term methods like pills and Injectables were more likely to experience failure than those using long term contraceptive methods. This could be because of inconsistency when using short term contraceptive methods which exposes women to increased failure rates. Similar findings were reported in $[2,12,13]$. Thus, information and counselling should be emphasized for short term method users to prevent failure.

At multivariate analysis, the most important factor associated with contraceptive failure was a woman's age. Women above 45 years of age had lower contraceptive failure rates than women below 40 years. This could be because women above 40 years are more likely than younger women to desire a permanent form of contraception[14,15]. Additionally, older women of reproductive age have lower rates of contraceptive failure than younger women and this could be explained by lower fecundity, less frequent sexual intercourse and higher compliance with contraceptive regimens as evidenced from previous studies [16].

This study used retrospective data from the DHS. The DHS follows a rigorous methodology employing a two-stage cluster randomized sampling. We used sample weights to provide estimates reported in this study. However, we acknowledge that contraceptive failure could best be studied using cohort studies. But this study provides further information about the current state of contraceptive failure among women of reproductive age in Uganda.

\section{Conclusion}

Our study found that there was a high burden of contraceptive failure among women of reproductive age in Uganda that and varies by socio-demographic characteristics. Traditional 
medRxiv preprint doi: https://doi.org/10.1101/2022.02.09.22270716; this version posted February 9, 2022. The copyright holder for this preprint (which was not certified by peer review) is the author/funder, who has granted medRxiv a license to display the preprint in perpetuity.

It is made available under a CC-BY 4.0 International license .

245 contraceptive methods including periodic abstinence and withdrawal are associated with higher

246 failure rates.

247 Declarations

248 Ethics approval and consent to participate

249 Ethical approval for the Demographic and Health Surveys is obtained from relevant research

250 ethical approval committees and research regulatory bodies before data collection. We obtained

251 permission to use the DHS survey datasets from the DHS program website. No personally

252 identifiable information is available in the used datasets.

253 Consent for publication

254 Not Applicable

255 Availability of data and materials

256 The datasets generated and/or analyzed during the current study are available in the Demographic

257 and Health Survey program website, https://hsprogram.com/data/available-datasets.cfm and can

258 be accessed after obtaining approval.

259 Competing interests

260 The authors declare that they do not have any competing interests.

261 Funding

262 The authors did not receive any funding for this work.

263 Authors' contributions

264 DBA and RKK conceived the study. DBA and RKK requested data from the DHS program 265 website, performed statistical analysis, interpretation of data and spearheaded the writing of the 266 manuscript. RKK and DBA discussed the relevant results. DBA, RKK, SK, FB and CGO 267 contributed to the writing of the manuscript, read, and approved the final manuscript. 
medRxiv preprint doi: https://doi.org/10.1101/2022.02.09.22270716; this version posted February 9, 2022. The copyright holder for this preprint (which was not certified by peer review) is the author/funder, who has granted medRxiv a license to display the preprint in perpetuity.

It is made available under a CC-BY 4.0 International license .

\section{Acknowledgements}

We thank the Demographic and Health Survey (DHS) program for granting us access to the DHS datasets used in this study

\section{References}

1. Starbird E, Norton M, Marcus R. Investing in family planning: Key to achieving the sustainable development goals. Glob Heal Sci Pract. 2016;4: 191-210. doi:10.9745/GHSP-D-15-00374

2. Ali MM, Cleland JG, Shah IH, Organization WH. Causes and consequences of contraceptive discontinuation: evidence from 60 demographic and health surveys. 2012.

3. Black KI, Gupta S, Rassi A, Kubba A. Why do women experience untimed pregnancies? A review of contraceptive failure rates. Best Pract Res Clin Obstet Gynaecol. 2010;24: $443-455$.

4. Polis CB, Bradley SEK, Bankole A, Onda T, Croft T, Singh S. Contraceptive Failure Rates in the Developing World: An Analysis of Demographic and Health Survey Data in 43 Countries. Contraception. 2016;94: 11-17. Available:

https://linkinghub.elsevier.com/retrieve/pii/S0010782416001037

5. Yazdkhasti M, Pourreza A, Pirak A, Abdi F. Unintended pregnancy and its adverse social and economic consequences on health system: a narrative review article. Iran J Public Health. 2015;44: 12.

6. Gipson JD, Koenig MA, Hindin MJ. The effects of unintended pregnancy on infant, child, and parental health: a review of the literature. Stud Fam Plann. 2008;39: 18-38.

7. Polis C, Bradley SEK, Bankole A, Onda T, Croft TN, Singh S. Contraceptive failure rates 
in the developing world: an analysis of demographic and health survey data in 43 countries. 2016.

8. Corsi DJ, Neuman M, Finlay JE, Subramanian S V. Demographic and health surveys: a profile. Int J Epidemiol. 2012;41: 1602-1613.

9. Samosir OB, Kiting AS, Aninditya F. Role of information and communication technology and women's empowerment in contraceptive discontinuation in Indonesia. J Prev Med Public Heal. 2020;53: 117.

10. Pradhan MR, Patel SK, Saraf AA. Informed choice in modern contraceptive method use: pattern and predictors among young women in India. J Biosoc Sci. 2020;52: 846-859.

11. Hilger DJ, Raviele KM, Hilgers TA. Hormonal contraception and the informed consent. Linacre Q. 2018;85: 375-384.

302 12. Bradley SEK, Schwandt H, Khan S. Levels, trends, and reasons for contraceptive discontinuation. DHS Anal Stud. 2009;20.

304 13. Westhoff CL, Heartwell S, Edwards S, Zieman M, Stuart G, Cwiak C, et al. Oral contraceptive discontinuation: do side effects matter? Am J Obstet Gynecol. 2007;196:

14. Alemayehu M, Belachew T, Tilahun T. Factors associated with utilization of long acting and permanent contraceptive methods among married women of reproductive age in Mekelle town, Tigray region, north Ethiopia. BMC Pregnancy Childbirth. 2012;12: 1-9.

310 15. Azmoude E, Behnam H, Barati-Far S, Aradmehr M. Factors affecting the use of longacting and permanent contraceptive methods among married women of reproductive age in East of Iran. Women's Heal Bull. 2017;4: 1-8.

313 16. Allen RH, Cwiak CA, Kaunitz AM. Contraception in women over 40 years of age. CMAJ. 
medRxiv preprint doi: https://doi.org/10.1101/2022.02.09.22270716; this version posted February 9, 2022. The copyright holder for this preprint (which was not certified by peer review) is the author/funder, who has granted medRxiv a license to display the preprint in perpetuity. 